\title{
Krise und Reform des Sozialstaates* \\ Eine wirtschaftsethische Analyse des ethischen Dilemmas der ökonomischen Sozialstaatsreformvorschläge
}

\author{
Christian BECKER UND FRANK JÖST ${ }^{* *}$
}

\section{Restructuring the Welfare State: A Business Ethics Analysis of the Ethical Dilemma of Economic Proposals}

This paper discusses the crucial role normative aspects play in the context of economic proposals for restructuring the welfare state. We identify the ethical and economic dimension of a welfare state reform, and analyse their complex relationship. In particular, we show that economic proposals for restructuring the welfare state do not refer to ethical aspects explicitly or in an encompassing manner. Rather, they implicitly contain certain value statements and, thus, lead to an implicit shift of social values. We show that this commingling of positive and normative statements is systematically grounded in the fundamentals of economics. The business ethics approach enables the analysis and identification of the normative aspects of economic proposals, and their integration into an explicit debate on social values, the economy, and the welfare state. From our analysis we draw methodological conclusions on the general inter-and transdisciplinary character of business ethics.

Keywords: Methodologie, Sozialstaatskrise, Sozialreform, Wirtschaftsethik.

\section{Einleitung}

Der deutsche Sozialstaat befindet sich in einer Krise. Dies ist Konsens in der gegenwärtigen öffentlichen Diskussion. Auch herrscht weitgehend Einigkeit über den unmittelbaren Zusammenhang dieser Krise mit einer ökonomischen Krise, die ebenfalls unisono konstatiert wird. Die Krise des Sozialstaates wird in Politik und Öffentlichkeit daher vorwiegend in ökonomischen Kategorien erfasst und diskutiert. Wirtschaftswissenschaftlichen Analysen und Lösungsvorschlägen kommt dementsprechend eine

Beitrag eingereicht am 11.07.2006; nach doppelt verdecktem Gutachterverfahren überarbeitete Fassung angenommen am 15.07.2007.

Für kritische Diskussion und konstruktive Kommentare danken wir Caroline Brett, Malte Faber, Reiner Manstetten, Thomas Petersen und zwei anonymen Gutachtern der $\mathbf{z} f \boldsymbol{w} \boldsymbol{u}$ sowie den Teilnehmern der 19. Tagung des Berliner Forums für Wirtschaftsetbik, der 19th Annual Conference of the European Business Ethics Network und des Seminars Denken-Entscheiden-Handeln: Etbik der Wirtschaft in Theorie und Praxis, insbesondere den Mitgliedern des Ronneburger Kreises.

** Dr. Christian Becker, Department of Philosophy, Dalhousie University, Halifax, NS, BH3 4P9, Canada, Tel.: +1-(902)494-3541, Fax: +1-(902)494-3518, E-Mail: c.u.becker@web.de, Web: www.philosophy.dal.ca.

PD Dr. Frank Jöst, Universität Heidelberg, Alfred-Weber-Institut für Wirtschaftswissenschaften, Grabengasse 14, D-69117 Heidelberg, Tel.: +49-(0)6221-54-2947, E-Mail: frank.joest@uni-hd.de. 
prominente Rolle zu. Sie beinhalten zumeist Teillösungen für jeweils bestimmte Bereiche der Sozialsysteme und thematisieren in erster Linie deren Effizienz und Transparenz (vgl. etwa Breyer et al. 2004, Zimmermann 2006).

Weder der Zusammenhang zwischen Problemen der Sozialsysteme und wirtschaftlichen Aspekten noch ein entsprechender Erkenntnisgewinn durch ökonomische Analysen sind zunächst von der Hand zu weisen. Wir behaupten allerdings, dass die Krise der Sozialsysteme nicht nur eine ökonomische ist, die mit ökonomischen Mitteln überwunden werden kann, sondern wesentlich auf eine fundamentale Krise der Gesellschaft verweist: Auf eine Krise unseres gesellschaftlichen Verständnisses von Wirtschaft und von sozialer Gerechtigkeit.

Diese fundamentale Krise wird in der gegenwärtigen Diskussion nicht explizit wahrgenommen und reflektiert. Hierzu fehlt insbesondere eine genaue Identifizierung der ökonomischen und ethischen Dimension des Problems der Krise des Sozialstaates sowie eine geeignete Verknüpfung ökonomischer Betrachtungen mit ethischen und politischen Reflexionen. Fraglich ist etwa, was wir unter dem Sozialstaat und seinen Aufgaben bzw. was wir unter sozialer Gerechtigkeit verstehen wollen. Wollen wir uns z. B. eher an einer angloamerikanischen Sozialstaatsidee der reinen Armutsbekämpfung orientieren oder an der in den skandinavischen Ländern traditionell verankerten Idee einer weitergehenden sozialen Umverteilung? Ebenso ist fraglich, wie wir Wirtschaft verstehen wollen. Welche Rolle soll dieser etwa im Selbstverständnis und in der Ausgestaltung der Gesellschaft zukommen? In diesem fundamentalen Sinne ist das Verständnis von sozialer Gerechtigkeit und von Wirtschaft ungeklärt, und in dieser Ungeklärtheit besteht die eigentliche Krise, die also wesentlich eine politisch-ethische ist (vgl. auch Sachße/Engelhardt 1990).

In der öffentlichen Debatte, aber auch insbesondere in wirtschaftswissenschaftlichen Beiträgen, tritt an die Stelle einer expliziten, fundamentalen ethisch-politischen Reflexion solcher Fragen vorwiegend ihre implizite Beantwortung. Diese ergibt sich quasi im Gefolge der ökonomischen Reformvorschläge, von denen viele auf die Implementierung einer angloamerikanischen Sozialstaatsidee hinauslaufen. Eine solche normative Entscheidung erscheint oftmals als zwingende Folge gegebener ökonomischer Notwendigkeiten. Die erforderliche grundlegende politisch-ethische Analyse wird so durch eine ökonomische substituiert, ohne den kategorialen Unterschied zwischen einer (wirtschafts)wissenschaftlichen und einer ethischen Problemstellung zu beachten.

Dass eine so zentrale gesellschaftliche Wertentscheidung auf diese Weise indirekt und unreflektiert vonstatten geht, verschärft das krisenhafte Moment an der Situation unserer Sozialsysteme. Hieraus resultieren Irritationen und Ängste bei den Menschen, die mit diesem impliziten fundamentalen ethischen Richtungswechsel konfrontiert werden, ohne dass eine explizite und bewusste öffentliche Debatte hierüber stattfindet. In der weit verbreiteten Skepsis und Ablehnung gegenüber den ökonomischen Reformvorschlägen kommt ein instinktives Bewusstsein von der Problematik der unreflektierten Vermischung ethischer Aspekte und ökonomischer Analyse zum Ausdruck.

Die explizite Untersuchung dieser Vermischung ist eine wirtschaftsethische Aufgabe und zentrales Thema dieses Papiers. Im Rahmen der Wirtschaftsethik kann die Frage nach der ethischen und ökonomischen Dimension des Problems integriert untersucht 
sowie das Verhältnis und die komplexe Verflechtung beider Dimensionen analysiert werden. Dies ist Voraussetzung für integrierte praktische Lösungsansätze, welche ethische und ökonomische Aspekte in adäquater Weise identifizieren und in die öffentliche Entscheidungsfindung einbringen.

Wir beginnen in Abschnitt 2 zunächst mit Überlegungen zur ethischen Dimension, indem wir den Begriff der sozialen Gerechtigkeit als zentralen ethischen Begriff der Sozialstaatesdebatte betrachten und die historische und kulturelle Gewachsenheit dieser Wertvorstellung betonen. In Abschnitt 3 analysieren wir exemplarisch die normativen Konnotationen ökonomischer Reformvorschläge und zeigen, dass diese implizit zumeist die Forderung nach einem angloamerikanischen Sozialstaatsmodell beinhalten. Eine kritische Reflektion hinsichtlich der Zulässigkeit solcher normativen Momente in der wissenschaftlichen Expertise führt uns in Abschnitt 4 zur Frage der Trennung von normativen Forderungen und positiver Analyse ökonomischer Zusammenhänge. Wir konstatieren die praktische Schwierigkeit einer solchen Trennung: Die Ökonomik basiert wesentlich auf normativen Grundlagen, die ihre Aussagen und Ergebnisse prägen. Insbesondere die ökonomische Politikberatung umfasst immer zweierlei: Die positive Beschreibung von ökonomischen Fakten, Voraussetzungen und Folgen einer jeweiligen Reform der Sozialsysteme und einen normativen Beitrag zu dieser Frage. In Abschnitt 5 schließen wir mit Folgerungen aus unserer Analyse hinsichtlich der Methodologie der Wirtschaftsethik als eines inter- und transdisziplinären Forschungsbereichs.

\section{Die ethische Dimension des Sozialstaates}

In Theorie und Praxis finden sich unterschiedliche Konzepte vom Sozialstaat. Differenzen bestehen zum einen hinsichtlich Aufgaben, institutioneller Ausgestaltung (der Rolle von Staat, Markt und anderen Institutionen), rechtlicher Implementierung, Finanzierung, Anspruchsgrundlagen, Höhe und Umfang von Leistungen etc., zum anderen hinsichtlich der zu Grunde liegenden Wertvorstellungen zur Frage, Was ist sozial bzw. sozial gerecht?' Je nach Kriterium lassen sich verschiedene Typen von Sozialstaaten unterscheiden (vgl. Titmuss 1974, Esping-Andersen 1990, Kersbergen 1995, Schmidt 2005).

Wir konzentrieren uns im Folgenden auf ein zentrales Unterscheidungsmerkmal: den zu Grunde liegenden sozialen Wertekonsens, d. h. die jeweils historisch-kulturell geprägten spezifischen Begriffe von ,sozial' bzw. ,sozialer Gerechtigkeit' in den einzelnen Gesellschaften. Diese ethische Dimension ist wesentliche Basis eines Sozialstaates. ${ }^{1}$ Für den Erfolg einer Reform desselben ist ihre Berücksichtigung unabdingbar.

Der Sozial- bzw. Wohlfahrtstaat gründet sich auf die Vorstellung, dass auf irgendeine Weise in die gesellschaftlichen Interaktionen regulierend eingegriffen werden muss. Er

$1 \quad$ Wir fokussieren auf den Begriff der Gerechtigkeit, weil diese traditionell als ethische Schlüsselkategorie für die Gestaltung des sozialen Miteinanders von Menschen gilt. Vgl. etwa schon Aristoteles (z. B. EN 1129b f.) oder auch Rawls (1999: 3): „Justice is the first virtue of social institutions, as truth is of systems of thought." Auch in der neueren sozialwissenschaftlichen Forschung zum Sozialstaat wird auf die zentrale Bedeutung des Begriffes der Gerechtigkeit hingewiesen (vgl. etwa Döring et al. 1995). 
setzt ein Ziel voraus, eine normative Idee bzw. Vorstellung davon, was ein guter oder gerechter Zustand der Gesellschaft ist (vgl. auch Esping-Andersen 2002). Der Begriff der sozialen Gerechtigkeit oder des Sozialen bezeichnet einen gesellschaftlichen Wert bzw. eine soziale Norm, die sich durch keine wissenschaftliche Analyse ableiten und implementieren lässt. Die Feststellung, dass etwas sozial gerecht oder ungerecht sei, ist keine Tatsachenbehauptung, sondern eine Wertung. Sie betrifft die Ebene des Moralischen und fällt damit in den Bereich der (normativen und deskriptiven) Ethik.

Die ethische Dimension des Sozialstaates ist vielschichtig und wirft eine Reihe von Fragen auf (vgl. etwa Koslowski/Føllesdal 1996, van Parijs 1996 oder Sachße/Engelhardt 1990). Insbesondere stellt die normative Begründung sozialer Gerechtigkeit oder auch des Sozialstaates insgesamt ein zentrales theoretisches Problem an der Schnittstelle von Ethik und Politischer Philosophie dar. Hier gibt es eine Reihe einflussreicher Konzepte, etwa aristotelische bzw. neo-aristotelische, deontologische, utilitaristische oder vertragstheoretische Ansätze (vgl. Kersting 2000). In Bezug auf Reformvorschläge zum Sozialstaat ist immer auch explizit zu klären und zu begründen, welche ethische Konzeption man auf der normativen Ebene zu Grunde legt. Es kann hier kein Primat eines bestimmten Ansatzes gelten. Die verschiedenen Aspekte der normativen Ansätze sind in ihrer Relevanz für die Debatte zur Sozialstaatsreform kritisch zu reflektieren.

Dem ursprünglichen Wortsinn nach bedeutet Ethik zunächst das Gewohnte. Das verweist darauf, dass soziale Werte im Allgemeinen nicht einfach abstrakt beschlossen und implementiert werden, sondern sich in einem historisch-kulturellen Kontext ausbilden und in diesen eingebettet sind. Gemeinsame Grundwerte einer Gesellschaft sind relativ beständig und ändern sich nur langsam. Sie sind wesentlicher Teil der kulturellen Identität einer Gesellschaft und ihrer nachhaltigen Entwicklung. Zum Ausdruck kommen diese nur zum Teil in formellen Strukturen, wie etwa im Grundgesetz der Bundesrepublik. Ein großer Teil der inhaltlichen präziseren Ausgestaltung von gesellschaftlichen Werten ist informeller Natur. Nur wenn das Selbstverständliche gesellschaftlicher Wertvorstellungen in Frage steht, wird eine normativ-ethische Reflexion derselben notwendig, und in einer Neuorientierung bedürfen Werte einer vernünftigen Begründung - etwa im Rahmen eines gesellschaftlichen Diskurses. Dies ist vor allem in politischen, sozialen, wirtschaftlichen oder kulturellen Umbruchzeiten der Gesellschaft der Fall.

Für eine Reform des Sozialstaates ist es also insbesondere wichtig, zu berücksichtigen, dass hierbei wesentliche gemeinschaftliche moralische Vorstellungen über soziale Gerechtigkeit betroffen sind, die in einen historisch-kulturellen Kontext eingebettet und von Gesellschaft zu Gesellschaft unterschiedlich sind. Zur Illustration der kulturellen Verschiedenheit gesellschaftlicher moralischer Überzeugungen hinsichtlich der sozialen Gerechtigkeit unterscheiden wir in Anlehnung an die Typisierung von EspingAndersen (1990: 26ff.) exemplarisch drei Sozialstaatsmodelle:

(1) Erstens das angloamerikanische Modell einer reinen Armutsbekämpfung. Dieses Modell des Sozialstaates ist in verschiedenen Varianten in Kanada, Australien und den USA verwirklicht. Wir reduzieren die vielfältigen Aspekte auf einige wesentliche normative Grundlagen: Primat individueller Freiheit und Eigenverantwortung vor dem Eingriff und der Fürsorge des Staates; Primat individueller 
Leistungsanreize und Leistungsbereitschaft vor jeder Umverteilung; Akzeptanz hoher Ungleichverteilungen in Einkommen und Besitz; Primat privater Wohltätigkeit vor Staatsfürsorge (vgl. auch Kaufmann 2003: 82ff.).

(2) Zweitens das konservative Sozialstaatsmodell einer weitgehenden Bewahrung von Einkommen und gesellschaftlichem Status. Ein solches hat Deutschland lange Zeit geprägt und stellt quasi den Status quo der gesellschaftlichen Wertorientierung dar, den jede Reform des bundesrepublikanischen Sozialstaates berücksichtigen muss. Auch hier seien einige zentrale normative Merkmale dieses Modells genannt: Konstitutiv ist die Idee einer bestimmten gesellschaftlichen Stellung und Würdigkeit der Person und damit verbundenen sozialen Rechten (vgl. Ritter 1989: 187; Sozialgesetzbuch, I, \$\$1-10); dementsprechend gilt insbesondere die Sicherung der gesellschaftlichen Stellung und des Einkommens als sozial gerecht (vgl. auch Kaufmann 2003: 304ff.).

(3) Drittens das skandinavische Modell einer weitergehenden sozialen Fürsorge und Umverteilung. Hier liegt die Idee einer Gleichheit aller individuellen Mitglieder der Gesellschaft unabhängig von Leistungsfähigkeit oder gesellschaftlichem Status zugrunde (vgl. Andersen 1984, Kaufmann 2003: 170f.). Daraus resultierend gelten eine stärkere Umverteilung und ein Ausgleich der durch Leistungsunterschiede entstandenen Einkommens- bzw. Vermögensdifferenzen als sozial gerecht und erwünscht. Es besteht eine geringere Akzeptanz von Einkommensunterschieden. Dem Staat wird eine starke Rolle hinsichtlich sozialer Dienste und Umverteilung zugesprochen (vgl. Kaufmann 2003: 163ff.).

Die Fragen ,Was ist sozial?' bzw. ,Was ist sozial gerecht?' erfahren also in verschiedenen Gesellschaften ganz unterschiedliche Antworten. Im angloamerikanischen etwa dominiert eine bestimmte Wertvorstellung von der individuellen Freiheit, die diese in erster Linie als ungehinderte individuelle Entfaltungs- und Leistungsmöglichkeit versteht. Die Marktwirtschaft wird hierbei als ein idealer Ort für diese Entfaltung angesehen. ${ }^{2}$ Die hieraus entstehenden Resultate, größeres individuelles Risiko und Verantwortung oder große Unterschiede im Einkommen, werden viel eher akzeptiert, als etwa im skandinavischen Modell, dem die Wertvorstellung von der Gleichheit der sozialen Rechte jedes Einzelnen unabhängig von seiner Leistung im Wirtschaftsprozess zu Grunde liegt (vgl. hierzu auch Haller 1986). Während im angloamerikanischen das Soziale in den Bereich der privaten moralischen Verpflichtung zur Hilfe in der Not zufällt und eine nachrangige Korrektur von Marktergebnissen durch Hilfe für die Schwachen darstellt, ist im skandinavischen und konservativen Modell das Soziale als originäre moralische Norm in der Gesellschaft implementiert und Ungleichheiten,

Einer der bedeutendsten Theoretiker dieser Sichtweise ist sicherlich Friedrich August von Hayek. Bei ihm erscheint der Markt als eine Struktur spontaner Ordnung, in welcher sich eine bestimmte Idee individueller Entfaltungsfreiheit verwirklicht (von Hayek 1994). Die Frage nach einer sozialen Gerechtigkeit in Bezug auf Marktergebnisse erscheint von Hayek unsinnig, da es sich hier um nicht beabsichtigte Ergebnisse einer spontanen Ordnung handelt (von Hayek 1981: 102f.). 
welche durch die Wirtschaftssphäre entstehen, sind entsprechend regelmäßig durch den Staat auszugleichen. ${ }^{3}$

Die verschiedenen Ausgestaltungen der Wohlfahrtsstaaten sind also nicht nur politisch-historische Pfadbesonderheiten bzw. institutionelle Eigenheiten, sondern verweisen letztlich auf wesentliche gesellschaftlich-kulturelle Unterschiede in Wertvorstellungen ${ }^{4}$ (vgl. Kaufmann 2003: 11), insbesondere in den Vorstellungen von sozialer Gerechtigkeit. Diese Feststellung bedeutet nicht, dass wir hier einen radikalen ethischen Relativismus postulieren. Wir konstatieren in Hinsicht auf die Frage nach der ethischen Dimension des Sozialstaates vielmehr, dass diese zwei Ebenen umfasst: Erstens die Ebene der historisch-kulturellen Ausbildung und Besonderheit von Wertvorstellungen und zweitens die Ebene der theoretischen, vernünftigen Begründung von Wertvorstellungen. Beide können nicht sinnvoll vollständig voneinander getrennt werden. Theoretischer Begründungsdiskurs und reale Ausbildung von Werten sind aufeinander zu beziehen. Eine umfassende Berücksichtigung der ethischen Dimension des Sozialstaates erfordert damit zugleich (a) eine explizite Reflexion auf der Ebene normativer ethischer Theorien bzw. Sozialstaatstheorien, (b) eine Untersuchung der jeweils konkreten Ausgestaltung gesellschaftlicher Wertvorstellungen und (c) eine geeignete Bezugsetzung dieser beiden Ebenen. ${ }^{5}$

Ökonomische Beiträge zur Reform des Sozialstaates berücksichtigen die ethische Dimension nicht auf diese umfassende Weise. Soweit überhaupt hierauf Bezug genommen wird, dann - ohne dies weiter zu reflektieren und begründen - auf normative vertragstheoretische Überlegungen in Anlehnung an Rawls. So versuchen zum Beispiel Breyer et al. (2004) das ethische Moment des Sozialstaates durch ein abstraktes vertragstheoretisches Gedankenexperiment zu erfassen. Dieser spezifische, individualistisch-rationale ethische Zugang kann jedoch gerade die tatsächliche Gegebenheit sozialer Werte in ihrer historisch-kulturellen Bestimmtheit und Unterschiedlichkeit, die wir als ein wesentliches konstituierendes Moment des Sozialstaates beschrieben haben, nicht einholen. Die ethische Frage scheint bei Breyer et al. (2004) analog zur ökonomischen Frage individualistisch-rational analysierbar und Leitlinien und Ziele der Reform so allgemeingültig formulierbar. Der historisch gewachsene gesellschaftliche Status quo wird gegenüber der abstrakten Norm negativ bewertet, als Besitzstandswahrung, als Zustand der überwunden werden muss (ebd.: 2). Dass das, was

3 Es gibt natürlich auch innerhalb einzelner Gesellschaften unterschiedliche Wertvorstellungen. Das Verhältnis kultureller Übereinstimmung und innergesellschaftlicher Divergenz ist jeweils gesondert zu analysieren (vgl. hierzu z. B. Pioch/Vobruba 1995).

$4 \quad$ Diese stehen auch in engem Zusammenhang mit der jeweiligen Geistesgeschichte. Vgl. etwa die zentrale Bedeutung von Hegels Denken für die Anfänge des deutschen Sozialstaates (siehe Ritter 1989: 67ff.) oder die Rolle von Empirismus, Liberalismus und Utilitarismus für das angloamerikanische Modell.

5 Vgl. hierzu auch Liebig/Lengfeld (2002), die Bedeutung, Möglichkeiten und Grenzen einer interdisziplinären Verknüpfung von normativer und empirischer Gerechtigkeitsforschung diskutieren. Es besteht im Übrigen nicht notwendig ein Widerspruch zwischen dem Anspruch auf allgemeine Begründung in der normativen Ethik und der spezifischen historisch-kulturellen Besonderheit gesellschaftlicher Werte. Dass es hier Möglichkeiten einer sinnvollen Verknüpfung gibt, demonstriert bereits die aristotelische Ethik. 
sozial bzw. sozial gerecht ist, sich gerade auch im Status quo und seiner inhärenten Dynamik bestimmt, kann eine solche Perspektive nicht in den Blick bekommen. ${ }^{6}$

In der Regel berücksichtigen ökonomische Beiträge jedoch die ethische Dimension des Sozialstaates überhaupt nicht explizit, sondern transportieren vielmehr unreflektiert (und implizit) eigene normative Positionen. Die weitere Analyse befasst sich ausführlich mit diesem Sachverhalt und seinen Ursachen.

\section{3. Ökonomische Reformvorschläge und die Frage nach der ökonomischen Dimension des Problems}

Es gibt eine Vielzahl von ökonomischen Analysen und Reformvorschlägen zum Thema Sozialstaat. Wir skizzieren im Folgenden beispielhaft einige Kernaspekte, die kennzeichnend für die Beiträge der Ökonomik zu dieser Thematik sind. Wesentlich ist zum einen die Idee der Effizienz. Ein Zustand ist effizient, wenn es nicht möglich ist, bei gegebener Faktorausstattung von einem Gut mehr zu produzieren ohne zugleich von einem anderen Gut weniger zu produzieren bzw. ein Zustand ist Pareto-effizient, wenn es nicht möglich ist, ein Individuum besser zu stellen, ohne zugleich ein anderes schlechter zu stellen. Mit anderen Worten, es werden in einem effizienten Zustand keine volkswirtschaftlichen Ressourcen verschwendet. Ökonomen gehen stets davon aus, dass ein effizienter Zustand einem nichteffizienten vorzuziehen sei. Dies beruht auf der Annahme, dass mehr immer besser ist, dass durch das Erreichen eines effizienten Zustandes der ,gesellschaftliche Kuchen' maximal groß ist und hier mehr verteilt werden kann als in einem ineffizienten Zustand.

Da die ökonomische Theorie zeigt, dass unter bestimmten Bedingungen der Markt zu effizienten Resultaten führt (Arrow 1951, Debreu 1959), wird im Hinblick auf die Effizienz für die Sozialsysteme in der Regel mehr Markt und Eigenverantwortung (Zimmermann 2006) bzw. mehr Wettbewerb (Breyer 2006: 118), also in diesem Sinne mehr Wirtschaft gefordert. Auf diesem theoretischen Fundament basieren etwa auch die Empfehlungen des Sachverständigenrates zur Begutachtung der gesamtwirtschaftlichen Entwicklung zur Reform der Sozialsysteme, z. B. die aktuellen Einlassungen zur Reform des Gesundheitswesens (Sachverständigenrat 2006/07: 216ff.).

Ist Effizienz also immer gut? Gilt damit für die Reform des Sozialstaates ein Primat der Effizienz? Gilt ein Primat des Marktes? Viele ökonomische Reformvorschläge suggerieren das und beinhalten so eine normative Komponente: „Die Wertschöpfung muss Priorität über die Frage bekommen, wie das Produzierte gerechter verteilt wird“ (Zimmermann 2006: 9). Eine solche Sicht beruht auf der Voraussetzung bestimmter Wertvorstellungen: Dass mehr Sozialprodukt immer besser ist als weniger und dass aus einem Mehr an Sozialprodukt immer auch mehr Solidarität und soziale Gerechtigkeit folgen (vgl. etwa Raffelhüschen 2006: 119). Damit beruhen Reformvorschläge, die die Effizienz des wirtschaftlichen Systems in den Vordergrund stellen implizit auf einer speziellen quantitativen Sicht einer Verteilungsgerechtigkeit. 
Im dynamischen Kontext ergeben sich zusätzliche Aspekte. Hier wird darauf abgehoben, dass Anreize im zeitlichen Verlauf zu mehr Wachstum führen. Hierzu gehören insbesondere Einkommensanreize, welche zu mehr Leistung motivieren (vgl. etwa Sinn et al. 2002; Schneider 2006: 73). Daher wird oft gefolgert, es sei sozial „nur noch eine Grundversorgung“" (ebd.) durch staatliche Institutionen sinnvoll. Denn zum einen ermöglicht dies Raum für Marktlösungen im Bereich des Sozialen, zum anderen schafft dies individuelle Anreize zu mehr Leistung und Eigenverantwortung. Im Bereich des Gesundheitswesens solle man sich etwa ,auf die medizinischen Notwendigkeiten“ (Zimmermann 2006: 16) beschränken, im Bereich der Bildung im Kern auf „Basiswissen’ (ebd.), oder im Bereich der Arbeitslosenversicherung auf eine Grundversorgung (vgl. Breyer et al. 2004: 3 und 15ff.), wie sie zum Teil bereits durch die Harz IV Gesetze umgesetzt ist.

Auf der Basis solcher dynamisch-ökonomischer Überlegungen ergeben sich häufig weitreichende Reformvorschläge für den Sozialstaat. In der Regel wird aus diesen Überlegungen hieraus eine deutliche Reduktion des Umfangs sozialstaatlicher Leistungen gefordert. Die in vielen ökonomischen Beiträgen vorgetragenen Reformvorschläge laufen somit implizit auf eine Übernahme des oben skizzierten angloamerikanischen Modells hinaus. ${ }^{7}$ Dies wird in der Regel mit ökonomischen Notwendigkeiten und wirtschaftswissenschaftlichen Einsichten und Erkenntnissen begründet. Die Ziele einer Reform werden so im Rahmen ökonomischer Überlegungen bestimmt. ${ }^{8}$ Soweit allerdings explizit oder implizit behauptet wird, solche normativen Reformziele seien in wirtschaftlichen Tatsachen begründet bzw. logisch abgeleitet, macht sich die wirtschafswissenschaftliche Expertise eines Sein-Sollen-Fehlschlusses schuldig (vgl. hierzu grundlegend Hume 1740/2000: 3.1.1.27), d. h. es werden normative Aussagen aus positiven Aussagen abgeleitet.

Es wird in vielen Fällen nicht deutlich, dass mit den ökonomischen Vorschlägen zugleich auch eine Reihe von normativen Voraussetzungen (nicht Folgerungen!), auf denen das angloamerikanische Modell beruht als neue Werteorientierung des deutschen Sozialstaates proklamiert werden. Angesichts dieser Vermischung von ökonomischen mit bestimmten normativen Aussagen, von ökonomischer und ethischer Ebene, stellt sich die Frage nach der eigenständigen ökonomischen Dimension des Problems.

$7 \quad$ Sinn et al. (2002: 19) konstatieren im Hinblick auf die Sozialhilfe sogar explizit: „Wenn man den Arbeitsmarkt im Bereich niedriger Einkommen in Deutschland wieder funktionsfähig machen möchte, gibt es kaum Alternativen zu einer grundlegenden Reform des sozialen Sicherungssystems, die in die Richtung des US-amerikanischen Modells geht."

$8 \quad$ Breyer et al. (2004) gehen anscheinend nicht so vor, sondern definieren zunächst Leitlinien und analysieren dann Reformalternativen im Hinblick auf die Kompatibilität mit diesen Leitlinien. Die Leitlinien sind jedoch vertragstheoretische Konstrukte, die nicht unabhängig von der ökonomischen Analyse sind: Erstens basieren Vertragstheorie und Ökonomik auf ähnlichen Grundannahmen über das Verhalten und die Rationalität des Menschen, seine individuelle Freiheit etc., zweitens werden einige Normen der Leitlinien unmittelbar aus ökonomischen Überlegungen bzw. Grundlagen abgeleitet, so etwa die Ablehnung der Gleichverteilung aus Anreiz- und Wachstumsüberlegungen sowie die Norm der Effizienz (vgl. ebd.: 11-14; siehe auch Abschnitt 2 oben). 


\section{Das ethische Dilemma der Ökonomik}

Wir scheinen hier nun bei der alten Frage des Werturteilstreites angelangt, nämlich welche Rolle Werturteile im Rahmen der Wirtschafts- und Sozialwissenschaften spielen. Einen historischen Höhepunkt dieser Diskussion im Verein für Socialpolitik im Jahre $1914^{9}$ nimmt die Position von Max Weber ein, welche das allgemeine moderne sozialwissenschaftliche Wissenschaftsideal in entscheidender Weise geprägt hat. Im Kern ist diese Position durch das Postulat der, Wertfreiheit' (vgl. Weber 1918/1988) ${ }^{10}$ der Sozialwissenschaften bestimmt, das eine „Scheidung von empirischer Feststellung und praktischer Wertung“" (ebd.: 497) fordert. ${ }^{11}$

Der naive Versuch, sich jeder normativen Wertung zu enthalten und eine rein positive (nur auf Tatsachen beruhende) wirtschaftswissenschaftliche Analyse und Beratung zu liefern, greift allerdings zu kurz und ist zum Scheitern verurteilt. Eine solche, die Weber'sche Position verkürzende Sicht, ist prominent von Robbins (1935: 147ff.) vertreten worden und hat nachfolgend das Selbstverständnis der Ökonomik stark geprägt. Obgleich normative und positive Ebene analytisch als kategorial verschieden getrennt werden können, sind sie in der Ökonomik praktisch komplex miteinander verbunden. Die moderne Ökonomik beruht auf einer Reihe normativer bzw. ethisch relevanter Voraussetzungen, die etwa in ihren spezifischen Rationalitätsannahmen, dem Effizienzbegriff, dem Marktideal usw., zum Ausdruck kommen (vgl. etwa Hausman/McPherson 2006, Ulrich 1997). ${ }^{12}$ Diese sind zudem eng verbunden mit bestimmten liberalen Grundwerten, etwa einem spezifischen Verständnis von individueller Freiheit oder auch außerwissenschaftlichen politischen Wertsetzungen, wie etwa dem heute weitgehend positiv betrachteten Wirtschaftswachstum.

$9 \quad$ Zu historischen Hintergründen und Gegenstand der Diskussion siehe Nau (1996). Hier findet sich auch der vollständige Text aller Diskussionsbeiträge.

Der hier zitierte Beitrag von Weber ist eine spätere, von ihm überarbeitete Fassung seiner Position. Für den Originalbeitrag für den Verein für Socialpolitik von 1913 siehe Nau (1996: 147186).

11 Hieran anknüpfend könnte man den oben skizzierten Sachverhalt der Vermischung ökonomischer und normativer Aspekte in wirtschaftswissenschaftlichen Reformvorschlägen als individuellen Fehler der jeweiligen Wissenschaftler sehen und werten. Weber (1918/1988: 498) konstatiert entsprechend, ,(...) dass man gerade unter dem Schein der Ausmerzung aller praktischen Wertungen ganz besonders stark, nach dem bekannten Schema: »die Tatsachen sprechen zu lassen", suggestiv solche hervorrufen kann [...und...] dass dies auf dem Katheder, gerade von Standpunkt der Forderung jener Scheidung aus, von allen Missbräuchen der allerverwerflichste wäre (...)“. Es erscheint allerdings unplausibel, dass die verbreitete Vermischung von positiven und normativen Aspekten sich als individuelle Fehler gleich einer Reihe renommierter Experten erklärt. Auch Verschwörungstheorien jeglicher Art sind wenig hilfreich. Wir gehen dagegen vielmehr davon aus, dass es systematische Schwierigkeiten bezüglich der Scheidung normativer und positiver Aspekte gibt, welche in der Konzeption der Ökonomik als Wissenschaft begründet sind. Weber (1918/1988) sieht das zwar im Prinzip, hat aber die Komplexität des Problems und die institutionellen Implikationen diesbezüglich unterschätzt.

12 In Bezug auf die Rationalitätsannahme hat schon Max Weber (1918/1988) diesen Umstand als ein notwendiges Merkmal der Sozialwissenschaften betont und seine Bedeutung im Rahmen seines Konzeptes des Idealtypus ausführlich diskutiert. 
Die wirtschaftswissenschaftliche Aussage etwa, dass Fragen der Effizienz und der Gerechtigkeit getrennt werden können, beruht schon auf einer Reihe normativer Voraussetzungen und gilt im Übrigen auch nur für den statischen Fall vollkommener Information. Im dynamischen Kontext, der für politische Fragen einer Sozialstaatsreform wesentlicher ist, wird oftmals gerade eine normative Verteilungsaussage zugunsten einer ineffizienten Allokation gemacht, die als Voraussetzung für größeres Wachstum gesehen wird: So werden etwa zeitlich befristete Monopole durch Patentschutz (die statische Ineffizienz bedeuten) als dynamische Wachstumsanreize verstanden. Ebenso gilt eine Ungleichverteilung von Einkommen im Hinblick auf Leistung etc. als dynamischer Anreiz zu Wirtschaftswachstum: „(...) die Umverteilung [hat] Grenzen, und sie sollte so organisiert werden, dass die Leistungsbereitschaft der Bürger möglichst wenig gehemmt wird" (Sinn et al. 2002: 19). Im Rahmen ökonomischer Analysen sind daher Aussagen über Verteilungsaspekte oft schon integriert.

Derartige Aussagen beruhen auf der Vorstellung eines Primates der Anreizwirkung sowie eines Primates individueller Leistungsbereitschaft und individueller Freiheit. Dahinter steht zum einen die Annahme, dass monetäre bzw. materielle Anreize für individuelle Leistungen und Entscheidungen ausschlaggebend sind und solche Momente wie Berufung (etwa bei Ärzten) oder Pflicht (etwa bei Beamten) keine wesentliche Rolle spielen (vgl. etwa auch Schneider 2006: 53). Zum anderen die Annahme, dass individuelle Freiheit in erster Linie in der ungehinderten Entfaltung der individuellen Leistungsmöglichkeit bestehe und Vorrang vor Solidarität habe. Aus dieser Perspektive besteht dann ein Widerspruch zwischen sozialer Umverteilung, welche diese Anreize vermindert, und wirtschaftlichem Wachstum, das gerade als eine Voraussetzung für soziale Gerechtigkeit gesehen wird. Dieser Widerspruch gründet sich nicht auf Tatsachen, sondern auf den zu Grunde liegenden Annahmen über das menschliche Verhalten und spezifischen (ökonomischen) Deutungen von Grundwerten, wie der individuellen Freiheit. ${ }^{13}$

Daher ist es auch nicht möglich, eine wertfreie Analyse der Bedingungen und Folgen bestimmter Sozialstaatsziele oder -reformen vorzunehmen, wie etwa Breyer et al. (2004) annehmen. Eine vollständige Trennung von Zielbestimmung auf gesellschaftlicher Ebene, und wirtschaftswissenschaftlicher Analyse der Mittel ist nicht möglich. Ein Vorgehen, das erst eine Ziel- bzw. Normenbestimmung vornimmt und dann analysiert, was diese ökonomisch bedeuten, inwiefern sich bestimmte Ziele umsetzen lassen und welche Konsequenzen sich ergeben werden, enthält in sich schon eine ethische Schwierigkeit. Denn auch bei einer Evaluation auf der Ebene der Mittel operiert die Ökonomik zwangsläufig auf einer bestimmten, ethisch relevanten Basis, die die Ergebnisse mit bestimmt. Wenn diese Basis im Widerspruch mit den normativen Voraussetzungen steht, auf denen die Zielvorgaben beruhen, kann sinnvoller Weise nur ein ethischer Disput über Werte geführt werden und nicht über rein positiv bestimmbare Voraussetzung, Folgen oder Umsetzungsmöglichkeiten der Zielvorgaben. Auf der wissenschaftlichen Ebene bleibt sonst ein scheinbar unlösbarer Konflikt bestehen. schichte des ökonomischen Denkens ist der Beitrag von Malthus (1798/1976: Ch. V) zur Frage der Armengesetzgebung in England. 
Es sind also zusammenfassend zwei Irrtümer hinsichtlich des Beitrages der Ökonomik zur Sozialstaatskrise und Sozialstaatsreform zu unterscheiden: Erstens der Irrtum, dass man aus einer rein positiven Analyse normative Reformziele ableiten könne (denn aus positiven Aussagen lassen sich logisch keine normativen Aussagen ableiten), zweitens der Irrtum, dass ein rein positiver wirtschaftswissenschaftlicher Beitrag zu Sozialstaatskrise und -reform möglich ist (denn alle ökonomischen Aussagen beruhen auf einer Reihe normativer Aussagen).

Korrekt wäre vielmehr, den wirtschaftswissenschaftlichen Beitrag im Feld der Politikberatung von vorne herein auch als einen normativen Beitrag zu verstehen. Denn die normativen Aussagen zu Reformzielen ergeben sich tatsächlich korrekt aus der ökonomischen Theorie, wenn man die entsprechenden normativen Prämissen in der Analyse bzw. Schlussfolgerung explizit und richtig berücksichtigt. (Aus normativen Prämissen lassen sich normative Aussagen ableiten). Man sollte also weder so tun, als ob sich ein wirtschaftswissenschaftlicher Reformvorschlag zwingend aus einer positiven Analyse der Wirtschaft ergäbe, noch überhaupt die Möglichkeit einer solchen rein positiven Analyse behaupten. Der adäquate Weg ist vielmehr, die der Analyse und den Reformvorschlägen zu Grunde liegenden normativen Prämissen explizit offen zu legen und als normativen Beitrag in die Zieldiskussion mit einzubringen. Dies überschreitet jedoch den engeren Bereich der Wirtschaftswissenschaften. Es ist ein komplexes Problem, dass weder zum primären Gegenstandsbereich der Wirtschaftswissenschaften gehört noch mit ihren Methoden zu behandeln ist. Hier bedarf es des Beitrages der Wirtschaftsethik.

\section{Fazit: Folgerungen für die Wirtschaftsethik und ihre Methodologie}

Das Verhältnis von Wirtschaft und sozialer Gerechtigkeit ist ein Gegenstand der Wirtschaftsethik. Ihre Aufgabe beschränkt sich hierbei nicht nur auf die Fragen: Welche Sozialstaatsidee ist kompatibel mit dem modernen wirtschaftswissenschaftlichen Begriff von Wirtschaft? Welches soziale Moralsystem ist optimal implementierbar unter den Bedingungen der modernen Wirtschaft? (vgl. Homann 1999; Homann/Lütge 2004: 65f.; Sinn et al. 2002) Diese Fragen setzen bereits ein Primat eines bestimmten Begriffes von Wirtschaft voraus, das selbst einer Klärung bedarf. Die Aufgabe der Wirtschaftsethik ist vielmehr eine allgemeine, nicht von vorne herein an ein bestimmtes Verständnis oder einen bestimmten Begriff von Wirtschaft gebundene Reflexion des Verhältnisses von Wirtschaft und Moral. Weder das Wirtschaftsverständnis und die Begrifflichkeiten der modernen Ökonomik noch eine bestimmte Ethikkonzeption sollten unreflektiert in einen wirtschaftsethischen Ansatz übernommen werden.

Jeder Begriff von Wirtschaft - insbesondere der moderne wirtschaftswissenschaftliche Begriff - stellt ein spezifisches, voraussetzungsreiches Verständnis von Wirtschaft dar, das insbesondere auf einer Reihe von normativen Annahmen beruht. In dieser Hinsicht gibt weder ein unbedingt gültiges Verständnis von Wirtschaft noch Gesetze der Wirtschaft im Sinne strenger Naturgesetze. Wirtschaft ist nur zum Teil eine gegebene Handlungsanforderung. Nur in unserer Naturabhängigkeit ist sie dies in einem strengen Sinne. Im Wesentlichen ist Wirtschaft ein gesellschaftlich gestaltetes System in einem historisch-kulturellen Kontext. Wir sind tatsächlich mit einem bestimmten 
Teilwertesystem konfrontiert, welches die Wirtschaft repräsentiert und das in einem Verhältnis zu anderen Wertvorstellungen einer Gesellschaft steht.

Diese Sicht auf Wirtschaft und Wirtschaftswissenschaft eröffnet wieder einen Spielraum für grundlegende wirtschaftsethische Fragen: Was für eine Wirtschaft will die Gesellschaft? Was für eine Rolle soll der Wirtschaft in der Gesellschaft zukommen? Oder einfach: Was ist eine gute Wirtschaft? Derartige Fragen sind solange sinnlos, wie Wirtschaft als ein faktisch Gegebenes, eine quasi unveränderliche gesetzliche Struktur aufgefasst wird, wie dies im Rahmen der Wirtschaftswissenschaften oder auch in Teilen von Politik und Öffentlichkeit, insbesondere unter dem Eindruck der Globalisierung, oft der Fall ist. Wir haben jedoch durch unseren Hinweis auf die normativen Elemente des modernen Wirtschaftsverständnisses verdeutlicht, dass nicht nur das gesellschaftliche Wirtschaftsverständnis ein Teilwertesystem der Gesellschaft repräsentiert, sondern insbesondere auch die Wirtschaftswissenschaften ein spezifisches, normativ basiertes Verständnis von Wirtschaft vermitteln. Oben genannte Fragen nach einer guten Wirtschaft sind demnach nicht nur zulässig, sie sind unseres Erachtens auch zentral für die Frage der Sozialstaatskrise und der Reform des Sozialstaates aus wirtschaftsethischer Perspektive.

Im Rahmen der allgemeinen wirtschaftsethischen Fragen nach dem Verhältnis von Wirtschaft und Moral ist daher Wirtschaft als ein Teilwertesystem neben anderen Wertesystemen der Gesellschaft zu betrachten und mit anderen Werten wie sozialer Gerechtigkeit in Bezug zu setzen. Es ist etwa explizit zu fragen: Wie verhalten sich Werte wie individuelle (ökonomische) Freiheit, Wachstum, Konsumentensouveränität, welche mit dem modernen wirtschaftswissenschaftlichen Wirtschaftsbegriff verbunden sind, zu Werten wie Solidarität, soziale Gerechtigkeit, Nachhaltigkeit etc.?

Die Analyse der aktuellen ökonomisch geprägten Debatte um die Sozialstaatskrise und der Entwicklung von Reformansätzen verdeutlicht schließlich einige zentrale methodologische Aspekte hinsichtlich der inter- und transdisziplinären Verfasstheit der Wirtschaftsethik. Es lassen sich zunächst vier verschiedene Zugangsebenen der wirtschaftsethischen Analyse identifizieren:

- Erstens die Ebene der Analyse der normativen Aspekte wirtschaftswissenschaftlicher Theorie. Im Einzelnen ist nicht einfach zu lokalisieren und zu bestimmen, welche ökonomischen Begriffe, Annahmen und Aussagen welche normativen Momente enthalten, auf welchen Werten beruhen oder welche ethischen Implikationen mit sich bringen. So ist beispielsweise eine genaue Analyse der Varianten der Rationalitätsannahme, des Effizienzbegriffes, des Marktideals, etc. notwendig. Diese Aufgabe erfordert eine Zusammenarbeit der Wirtschaftsethik mit der Wissenschaftstheorie der Ökonomik (philosophy of economics).

- Zweitens die Ebene normativer ethischer Ansätze und die Frage nach deren jeweiliger Relevanz für die konkrete wirtschaftsethische Fragestellung. Dies beinhaltet den Bezug der Wirtschaftsethik als angewandter Ethik zur philosophischen Ethik und zu den etablierten normativen Ethikkonzeptionen.

- Drittens die Ebene der Analyse bestehender gesellschaftlicher Wertvorstellungen und ihrer historischen und kulturellen Besonderheiten. Hier geht es etwa 
um die Identifizierung von Gerechtigkeitsvorstellungen und Begriffen des Sozialen oder anderen Werten in einem konkreten gesellschaftlichen Kontext. Es geht aber ebenso auch um die Identifizierung unterschiedlicher Wirtschaftsverständnisse bzw. Vorstellungen von Wirtschaft in einer Gesellschaft. Dies erfordert eine Verknüpfung der Wirtschaftsethik mit anderen (empirischen) Sozialund Kulturwissenschaften, wie etwa Soziologie, Politik oder Ethnologie.

- Viertens geht es schließlich um eine geeignete Bezugsetzung der normativen Voraussetzungen der ökonomischen Theorie mit ethischen Begründungskonzepten und gesellschaftlichen Wertvorstellungen sowie um die Frage nach Kompatibilität und Widersprüchen. Diese Reflexionen sind schließlich explizit in einen öffentlichen politisch-ethischen Diskurs einzubringen. Die Wirtschaftsethik kann hierdurch den Weg für eine umfassende Debatte zum Verständnis von Wirtschaft und sozialen Werten sowie zur Bedeutung von Wirtschaft im Rahmen von gesellschaftlicher Ordnung und Zielsetzung eröffnen. In dieser Hinsicht kommt der Wirtschaftsethik auch eine vermittelnde Rolle zwischen Wissenschaft und öffentlicher Debatte zu.

Die ersten beiden Ebenen betreffen den Bezug der Wirtschaftsethik zu den Reflektionsebenen Ökonomik und Ethik, Ebene 3 den Bezug zu den Gegenstandsbereichen Wirtschaft und Moral, und Ebene 4 die Bezugsetzung der genannten Ebenen sowie die Verbindung wissenschaftlicher Analyse mit dem gesellschaftlichen Diskurs. Dies zeigt, dass die Wirtschaftsethik ein komplexer, inter- und transdisziplinärer Forschungsbereich ist. Die dargestellten Analyseebenen erfordern den Einbezug von Kenntnissen und Methoden verschiedener Disziplinen in den Rahmen wirtschaftsethischer Analyse. Das bedeutet eine vielfältige interdisziplinäre Zusammenarbeit, die im Hinblick auf die beteiligten Disziplinen jeweils auf den konkreten Untersuchungsgegenstand anzupassen ist. Dieses interdisziplinäre Moment der Wirtschaftsethik wurde in verschiedenen methodologischen Arbeiten erkannt und angesprochen (vgl. De George 1987, Shaw 1996, Spence 1998).

Unsere Analyse verweist jedoch auf zwei methodologische Besonderheiten: Zum einen zeigt sich die zentrale Bedeutung einer Verknüpfung der Wirtschaftethik mit der Wissenschaftstheorie der Ökonomik. Diese Verknüpfung ist für die Wirtschaftsethik von grundlegender methodologischer Bedeutung, da eine umfassende Untersuchung des Verhältnisses von Wirtschaft und Moral eine kritische Auseinandersetzung mit dem Wirtschaftsverständnis der modernen Wirtschaftswissenschaften und dessen normativen Aspekten erfordert. In Bezug auf eine solche Verknüpfung besteht noch großes Potential für die weitere wirtschaftsethische Forschung. Zum anderen kommt der Wirtschaftsethik ein transdisziplinäres Moment zu, insofern sie theoretische und praktische Aspekte eines Problems miteinander in Bezug setzt und hiermit einen Beitrag zu öffentlichem Diskurs und Entscheidungsfindung leistet. Etwa indem sie Konflikte und Widersprüche, die zwischen wissenschaftlichen Analysen und gesellschaftlichen Reaktionen bestehen auf dahinter liegende Wertekonflikte zurückführt, und so z. B. das Problem der Sozialstaatskrise als ethisches und ökonomisches Problem umfassend diskutierbar macht. Hinsichtlich einer weitergehenden methodologischen Diskussion dieses Aspektes empfiehlt sich eine Verbindung mit der bestehenden Forschung zur Transdisziplinarität (vgl. etwa Thompson-Klein et al. 2001). 
Die wirtschaftethische Analyse dient letztlich auch den Sozialwissenschaften, etwa der Ökonomik, und ihrer Erkenntnisfunktion und Rolle im Rahmen von Gesellschaft und Politikberatung. So kann etwa der wirtschaftswissenschaftliche Beitrag zur Reformdebatte des Sozialstaates erst dann angemessen Berücksichtigung finden und umfassend fruchtbar gemacht werden, wenn er in seiner normativen Dimension explizit erfasst und eingebracht wird. Erst dies eröffnet die Möglichkeit, das Verständnis von Wirtschaft und ihrer Bedeutung im Rahmen der Gesellschaft auch jenseits des wirtschaftswissenschaftlichen Verständnisses von Wirtschaft umfassend zu diskutieren. Wirtschaft wird so nicht unreflektiert als gegebene Bedingung gesellschaftlichen Handelns angenommen, sondern kann als Aspekt gesellschaftlicher Ziel- und Wertsetzungen in einer umfassenden Ziel- und Wertedebatte in ihrer Bedeutung im Verhältnis zu anderen gesellschaftlichen Zielen und Werten explizit reflektiert werden.

\section{Literaturverzeichnis}

Andersen, B. R. (1984): Rationality and Irrationality of the Nordic Welfare State, in: Daedalus 113, 109-139.

Aristoteles (1991): Die Nikomachische Ethik. Übersetzt u. herausgegeben v. O. Gigon, München.

Arrow, K. J. (1951): An Extension of the Basic Theorems of Classical Welfare Economics, in: Neyman, J. (Ed.): Proceedings of the Second Berkeley Symposium on Mathematical Statistics and Probability, Berkeley, 507-532.

Breyer, F. (2006): Gesundheitsreform: Solidarität und Eigenverantwortung stärken, in: Zimmermann, K. F. (Hrsg): Deutschland - was nun? Reformen für Wirtschaft und Gesellschaft, München, 103-118.

Breyer, F./ Franz, W./ Homburg, S./ Schnabel, R./ Wille, E. (2004): Reform der sozialen Sicherung, Berlin.

Debreu, G. (1959): Theory of Value. An Axiomatic Analysis of Economic Equilibrium, New York.

De George, R. T. (1987): The Status of Business Ethics: Past and Future, in: Journal of Business Ethics 6(3), 201-11.

Döring, D./ Nullmeier, F./ Pioch, R./ Vobruba, G. (1995): Gerechtigkeit im Wohlfahrtsstaat, Marburg.

Esping-Andersen, G. (1990): The Three Worlds of Welfare Capitalism, Princeton.

Esping-Andersen, G. (2002): Towards the Good Society, Once Again?, in: Andersen, G. (Ed.): Why We Need a New Welfare State, Oxford.

Haller, M. (1986): Die Legitimation sozialer Ungleichheit im Wohlfahrtsstaat, in: Journal für Sozialforschung 26, 443-68.

Hayek, F. A. von (1981): Recht, Gesetzgebung und Freiheit, Bd. 2: Die Illusion der sozialen Gerechtigkeit. Landsberg am Lech.

Hayek, F. A. von (1994): Grundsätze einer liberalen Gesellschaftsordnung, in: Freiburger Studien. Gesammelte Aufsätze, 2. Aufl., Tübingen, 108-125.

Hausman, D. M./ McPherson, M. S. (2006): Economic Analysis, Moral Philosophy and Public Policy, 2nd edition, Cambridge. 
Homann, K. (1999): Zur Grundlegung einer modernen Gesellschafts- und Sozialpolitik. Das Problem der „sozialen Ordnung“, in: Blum, H. (Hrsg.): Soziale Marktwirtschaft im nächsten Jahrtausend, Stuttgart, 119-148.

Homann, K./ Lütge, C. (2004): Einführung in die Wirtschaftsethik, Münster.

Hume, D. (1740/2000): A Treatise of Human Nature, Oxford.

Kaufmann, F.-X. (2003): Varianten des Wohlfahrtsstaates. Der deutsche Sozialstaat im internationalen Vergleich, Frankfurt a. M.

Kersbergen, K. (1995): Social Capitalism. A Study of Christian Democracy and the Welfare State, London, New York.

Kersting, W. (Hrsg.) (2000): Politische Philosophie des Sozialstaates, Weilerwist.

Kliemt, H. (2000): Keine Theorie der Gerechtigkeit, in: Neumann, U./ Schulz, L. (Hrsg.): Verantwortung in Recht und Moral. Referate der Tagung der Deutschen Sektion der Internationalen Vereinigung für Rechts- und Sozialphilosophie vom 2. bis zum 3. Oktober 1998 in Frankfurt a. M., (ARSP Beiheft 74), Stuttgart, 217-228.

Koslowski, P./ A. Follesdal (Eds.)(1996): Restructuring the Welfare State. Theory and Reform of Social Policy, Berlin.

Liebig, S./ Lengfeld, H. (Hrsg.) (2002): Interdisziplinäre Gerechtigkeitsforschung. Zur Verknüpfung empirischer und normativer Perspektiven, Frankfurt a. M., New York.

Malthus, T. R. (1798/1976): An Essay on the Principle of Population. Ed. Philip Appleman, New York, London.

Nau, H. H. (1996): Der Werturteilsstreit. Die Äußerungen zur Werturteilsdiskussion im Ausschuß des Vereins für Sozialpolitik (1913), Marburg.

Parjis, P., van (1996): On the Moral Foundations of the Welfare State. Three Research Programmes, in: Koslowski, P./ Føllesdal, A. (Eds.): Restructuring the Welfare State. Theory and Reform of Social Policy, Berlin, 383-392.

Pioch, R./ Vobruba, G. (1995): Gerechtigkeitsvorstellungen im Wohlfahrtsstaat. Sekundäranalyse empirischer Untersuchungen zur Akzeptanz wohlfahrtsstaatlicher Maßnahmen, in: Döring, D./ Nullmeier, F./ Pioch, R./ Vobruba, G. (Hrsg.): Gerechtigkeit im Wohlfahrtsstaat, Marburg, 114-165.

Raffelbüschen, B. (2006): Generationengerechtigkeit: Das Beispiel Pflegeversicherung, in: Zimmermann, K. F. (Hrsg): Deutschland - was nun? Reformen für Wirtschaft und Gesellschaft, München, 119-130.

Rawls, J. (1999): A Theory of Justice. Revised edition, Cambridge.

Ritter, G. A. (1989): Der Sozialstaat. Entstehung und Entwicklung im internationalen Vergleich, München.

Robbins, L. (1935): An Essay on the Nature and Significance of Economic Science, 2nd edition, London.

Sachße, C./ Engelhardt, H. T. (Hrsg.) (1990): Sicherheit und Freiheit. Zur Ethik des Wohlfahrtsstaates, Frankfurt a. M.

Sachverständigenrat (2006/07): Widerstreitende Interessen - Ungenutzte Chancen. Jahresgutachten 2006/07 des Sachverständigenrats zur Begutachtung der gesamtwirtschaftlichen Entwicklung, Berlin.

Shaw, W. H. (1996): Business Ethics Today: A Survey, in: Journal of Business Ethics 15(5), 489-500.

Schmidt, M. G. (2005) Sozialpolitik in Deutschland. Historische Entwicklung und internationaler Vergleich, 3. Aufl., Wiesbaden. 
Schneider, H. (2006): Arbeitsmarkt: Rezepte gegen die unheilige Allianz von Politik und Tarifkartellen gefragt, in: Zimmermann, K. F. (Hrsg): Deutschland - was nun? Reformen für Wirtschaft und Gesellschaft, München, 43-74.

Sinn, H.-W./ Holzner, C./ Meister, W./ Ochel, W./ Werding, M. (2002): Aktivierende Sozialhilfe. Ein Weg zu mehr Beschäftigung und Wachstum, Ifo Schnelldienst 9/2002, München.

Spence, L. J. (1998): On Effective Interdisciplinary Alliances in European Business Ethics Research - Discussion and Illustration. in: Journal of Business Ethics 17(9/10), 10291044.

Thompson-Klein J./ Grossenbacher-Mansuy, W./ Häberli, R./ Bill, A./ Scholz, R. W./ Welti, M. (Eds.) (2001): Transdisciplinarity: Joint Problem Solving among Science, Technology, and Society. An Effective Way for Managing Complexity, Basel u. a.

Titmuss, R. M. (1974): Social Policy. An Introduction, London.

Ulrich, P. (1997): Integrative Wirtschaftsethik. Grundlagen einer lebensdienlichen Ökonomie, Bern u. a.

Weber, M. (1918/1988): Der Sinn der »Wertfreiheit« der soziologischen und ökonomischen Wissenschaften, 1917, in: Winckelmann, J. (Hrsg): Max Weber. Gesammelte Aufsätze zur Wissenschaftslehre, Tübingen, 489-540.

Zimmermann, K. F. (Hrsg.) (2006): Deutschland - was nun? Reformen für Wirtschaft und Gesellschaft, München. 